\title{
Neoplasia maligna de mama: conhecimento de estudantes de uma Universidade Federal do Nordeste
}

\author{
Malignant breast cancer: knowledge of students from a Federal University in the Northeast
Cáncer de mama maligno: conocimiento de estudiantes de una Universidad Federal en el Noreste

Thayse Luana Faria Costa Ramos ${ }^{1}$, Amuzza Aylla Pereira dos Santos ${ }^{1 *}$, Marianny Medeiros de Moraes $^{1}$, Maria Elisângela Torres de Lima Sanches ${ }^{1}$, Wanderlei Barbosa dos Santos ${ }^{1}$, José Augustinho Mendes Santos ${ }^{1}$, Larissa de Morais Teixeira ${ }^{1}$.

\section{RESUMO}

Objetivo: Descrever o nível de conhecimento de estudantes de uma universidade federal do Nordeste sobre neoplasia maligna da mama. Métodos: Trata-se de uma pesquisa de caráter descritivo, exploratório e quantitativa. A pesquisa foi realizada com discentes dos cursos de Enfermagem, Farmácia, Odontologia, Serviço Social, Geografia e Matemática. Os dados foram coletados após aprovação do projeto pelo Comitê de Ética em Pesquisa sob o ํo 65966217.6.0000.5013. Resultados: O estudo evidenciou quanto ao conhecimento adquirido dentro da universidade, onde só o curso de Enfermagem possui estudantes que se dizem informadas sobre o assunto. Conclusão: Foi identificado que a maioria das estudantes recebeu informações a respeito do tema, porém não foi a universidade que proporcionou este conhecimento. Neste contexto, é imprescindível repensar as práticas de educação e propagação do conhecimento, além de ser imprescindível que a universidade invista em meios de transformação através de estratégias que levem à promoção da saúde e formem cidadãos que serão propagadores de conhecimento, além de democratizar o acesso à informação no meio universitário.

Palavras-chave: Neoplasia de mama, Enfermagem, Estudantes.

\begin{abstract}
Objective: To describe the level of knowledge of students of a federal university in the Northeast about malignant breast cancer. Methods: This is a descriptive, exploratory and quantitative research. The research was carried out with students from the Nursing, Pharmacy, Dentistry, Social Work, Geography and Mathematics courses Data were collected after approval of the project by the Research Ethics Committee under number 65966217.6.0000.5013. Results: The study showed the knowledge acquired within the university, where only the Nursing course has students who say they are informed about the subject. Conclusion: It was identified that the majority of university students had information on the subject, but it was not the university that provided this knowledge. In this context, it is essential to rethink the practices of education and knowledge propagation, in addition to it being essential that the university invests in means of transformation through strategies that lead to health promotion and form citizens who can disseminate knowledge, in addition to democratizing access to information in the university environment.
\end{abstract}

Keywords: Breast neoplasms, Nursing, Students.

\section{RESUMEN}

Objetivo: Objetivo describir el nivel de conocimiento de estudiantes de una universidad federal del Nordeste sobre el cáncer de mama maligno. Métodos: Se trata de una investigación descriptiva, exploratoria y cuantitativa. La investigación se realizó con estudiantes de los cursos de Enfermería, Farmacia, Odontología, Trabajo Social, Geografía y Matemáticas. Los datos fueron recogidos para la aprobación del proyecto por el Comité de Ética en Investigación con el № 65966217.6.0000.5013. Resultados: El estudio mostró los

${ }^{1}$ Universidade Federal de Alagoas (UFAL), Maceió - AL. *E-mail: amuzza.santos@gmail.com 
conocimientos adquiridos dentro de la universidad, donde solo el curso de Enfermería cuenta con estudiantes que dicen estar informados sobre el tema. Conclusión: Se identificó que la mayoría de los estudiantes recibieron información sobre el tema, por lo que no fue la universidad la que brindó este conocimiento. En este contexto, es imprescindible repensar las prácticas de educación y difusión del conocimiento, además de ser imprescindible que la universidad invierta en medios de transformación a través de estrategias que conduzcan a la promoción de la salud y formen ciudadanos que serán propagadores del conocimiento, además de democratizar el acceso a la información en el ámbito universitario.

Palabras clave: Neoplasias de la mama, Enfermería, Estudiantes.

\section{INTRODUÇÃO}

As incidências dos casos de câncer de mama apresentam taxas de incidência que diferenciam quase quatro vezes entre as regiões do mundo, de 27 por 100.000 na África Central e na Ásia Oriental a 96 por 100.000 na Europa Ocidental, e tendem a ser elevadas nos países com maior desenvolvimento. É a causa mais frequente de morte por neoplasia maligna em mulheres nas regiões menos desenvolvidas (324.000 mortes, $14,3 \%$ do total) e a segunda causa de morte por neoplasia maligna nas regiões com desenvolvimento maior (198.000 mortes, 15,4\%), atrás da neoplasia maligna de pulmão. As taxas de incidência avançam em todos os países, salvo em alguns países que possuem renda elevada (JEMAL A, et al., 2014; BARTA SK, et al., 2015).

No Brasil, a neoplasia da mama corresponde a $22 \%$ dos novos casos a cada ano, tendo um nível elevado de mortalidade provavelmente por ter o diagnóstico tardio e em estágio avançado. $O$ aparecimento da neoplasia maligna da mama é comum a partir dos 40 anos, mas acomete principalmente a faixa etária de 50 a 69 anos e ocorrendo raramente antes dos 35 anos. No triênio 2020-2022, espera-se 62.280 novos casos de neoplasia maligna da mama, tendo um risco de 61,61 casos a cada 100 mil mulheres (STEWARD BW e WILD CP, 2014; INCA, 2015; INCA, 2019).

Porém, mesmo com o avanço nos campos de prevenção e de tratamento, a neoplasia maligna da mama continua como principal causa de morte entre mulheres no Brasil, crescendo em 17,7\% na taxa de mortalidade em 20 anos, levando a 13.345 óbitos em 2011, segundo o Instituto Nacional do Câncer (INCA) (BARRETONETO NJS, et al., 2014).

É um tipo de neoplasia maligna multifatorial, tendo fatores genéticos e ambientais contribuindo para o seu aparecimento. Os fatores de risco que contribuem para a neoplasia maligna da mama envolvem fatores reprodutivos "(menarca precoce, nuliparidade, idade maior do que 30 anos na primeira gravidez, uso de contraceptivos hormonais de alta dose, menopausa tardia e terapia de reposição hormonal)", mulheres com idade avançada, elevada densidade do tecido mamário e histórico de câncer na família (PROLLA CMD, et al., 2015; OLIVEIRA MF, et al., 2016).

A neoplasia maligna da mama é uma doença temida pelas mulheres. Frequentemente está associada à mutilação física, como a mastectomia total ou parcial, alterações no estilo e na qualidade de vida. Mesmo com o avanço tecnológico na saúde em relação ao tratamento e do aumento de informações veiculadas nos meios de comunicação e em campanhas, como é o caso do outubro rosa, as taxas de neoplasia mamária são grandes, certamente porque a doença ainda é diagnosticada em estágios avançados (GARCIA SN, et al., 2015).

No Brasil, a neoplasia maligna é uma patologia crítica complicando nos últimos anos em consequência do envelhecimento da população nos países subdesenvolvidos. É uma doença diferenciada de outras enfermidades crônicas em decorrência de sua patologia que pode ocasionar deformação, dor e mutilações, além de alta repercussão psicológica, levando a sentimentos negativos desde o instante do diagnóstico (LEITE MAC, et al., 2015).

Além disso, existe um protocolo para detecção da neoplasia maligna da mama que é recomendado pelo Ministério da Saúde e engloba o exame clínico anual para mulheres assintomáticas entre 40 e 50 anos e a mamografia bianual para mulheres entre 50 e 69 anos. As recomendações para mulheres com risco elevado 
para desenvolvimento da neoplasia maligna são estabelecidas menos claramente no Brasil, porém o Exame Clínico das Mamas (ECM) e a Mamografia Anual (MG) têm sido sugeridos a partir dos 35 anos de idade. Não existem evidências que sustentem o Autoexame das Mamas (AEM) como um método isolado para a detecção precoce da neoplasia maligna da mama (PROLLA CMD, et al., 2015).

Para que ocorra a detecção precoce da neoplasia maligna da mama o Ministério da Saúde promove campanhas nacionais de prevenção veiculadas nos meios de comunicação. Porém, os casos de neoplasia maligna da mama em mulheres jovens que obtêm o diagnóstico tardio, podem ser ocasionados pela falta de rastreamento adequado e dificuldade de leitura e interpretação dos resultados mamográficos devido à alta densidade mamária. Outra questão que pode contribuir "é a falsa percepção, por muitos profissionais de saúde, de que mulheres jovens não possuem risco de desenvolver neoplasia maligna, desvalorizando sinais e sintomas iniciais da doença" (PINHEIRO AB, et al., 2013; MIGOWSKI A, et al., 2018).

Em razão desses aspectos, o estudo justifica-se pelo fato de mulheres jovens estarem adoecendo por não reconhecerem os sinais e sintomas, bem como a não realização dos exames preventivos para a neoplasia maligna da mama. A universidade por ser um local de aprendizagem e socialização das informações para construção do saber pode contribuir orientando as estudantes a compreenderem o quanto a descoberta precoce pode ajudar no processo de tratamento e cura para este agravo. Diante do exposto, o presente estudo tem como objetivo: descrever o nível de conhecimento de estudantes de uma universidade federal do Nordeste sobre neoplasia maligna da mama.

\section{MÉTODOS}

Trata-se de um estudo descritivo, exploratório, com abordagem quantitativa. A pesquisa foi realizada numa Universidade Federal, em uma capital do Nordeste brasileiro.

A pesquisa foi realizada em seis unidades acadêmicas, a saber: cursos de Enfermagem, Farmácia, Odontologia, Serviço Social, Geografia e Matemática, escolhidos aleatoriamente para participar do estudo. Considerando que não apenas estudantes de cursos da área da saúde têm a possibilidade de acesso às informações pertinentes à temática do estudo, os cursos foram escolhidos englobando as demais áreas, como exatas e humanas. Além disso, partiu-se da hipótese que todas as estudantes têm o direito à informação e possuem probabilidade de serem acometidas pela neoplasia maligna da mama.

Participaram da pesquisa todas as estudantes que durante o período da coleta de dados atenderam aos seguintes critérios de inclusão: possuir idade igual ou superior a 18 anos e estar matriculada regulamente no curso pesquisado. Já os critérios de exclusão basearam-se em estudantes que estivessem em tratamento de neoplasias da mama e que estivessem passando por algum estresse emocional e/ou debilidade que a impossibilitasse de participar da pesquisa. Todas as estudantes que atenderam aos critérios de inclusão foram convidadas a participar da pesquisa e assinaram o Termo de Consentimento Livre e Esclarecido (TCLE).

A pesquisa foi realizada no período de junho a agosto de 2017 por meio de um questionário semiestruturado onde continha dados sociodemográficos e do objeto de estudo. As variáveis avaliadas estão apresentadas no Quadro 1.

Quadro 1 - Variáveis analisadas no instrumento de coleta de dados.

\begin{tabular}{|c|c|}
\hline Finalidades & Variáveis analisadas \\
\hline $\begin{array}{c}\text { Avaliar o perfil sóciodemográfico das } \\
\text { estudantes }\end{array}$ & Idade; cor e estado civil \\
\hline $\begin{array}{c}\text { Conhecimento e prática a respeito da } \\
\text { neoplasia maligna da mama }\end{array}$ & $\begin{array}{c}\text { Informações sobre neoplasia maligna da } \\
\text { mama e locais dessas informações }\end{array}$ \\
\hline $\begin{array}{c}\text { Conhecimento sobre os métodos de } \\
\text { detecção precoce da neoplasia maligna da } \\
\text { mama }\end{array}$ & Informações sobre o autoexame das mamas \\
\hline $\begin{array}{c}\text { Informações adquiridas dentro da } \\
\text { universidade }\end{array}$ & $\begin{array}{c}\text { Informações sobre neoplasia maligna da } \\
\text { mama dentro da unidade acadêmica }\end{array}$ \\
\hline
\end{tabular}

Fonte: Ramos TLFC, et al., 2021. 
O tamanho da amostra foi estimado em 206 estudantes dos cursos propostos, considerando o percentual mínimo de 80\%, o erro amostral de 5\%, a população de 1253 estudantes que compõem os cursos pesquisados e o nível de confiança em 95\%. Foi utilizada calculadora eletrônica disponível na internet no URL: http://www.publicacoesdeturismo.com.br/calculoamostral/.

Os dados foram analisados no programa Statistical Package for Social Science (SPSS), modelo língua portuguesa (versão 20) e foi utilizada a estatística descritiva para o cálculo da frequência absoluta (n) e do percentual de cada variável (F\%). Ademais, foram transferidos para o programa Microsoft Excel para geração dos gráficos e para Word para elaboração das tabelas.

Os dados foram coletados após aprovação do projeto pelo Comitê de Ética em Pesquisa (CEP) da Universidade Federal de Alagoas sob o nํ 65966217.6.0000.5013.

\section{RESULTADOS}

Participaram da pesquisa 206 estudantes de uma Universidade Federal do Nordeste. As características sociodemográficas das estudantes estão descritas na Tabela 1.

Tabela 1 - Características sociodemográficas de estudantes de uma Universidade Federal do Nordeste.

\begin{tabular}{|c|c|c|}
\hline Variáveis & (n) & (\%) \\
\hline \multicolumn{3}{|l|}{ Faixa etária } \\
\hline $18-22$ & 119 & $57,77 \%$ \\
\hline $23-27$ & 64 & $31,07 \%$ \\
\hline $28-32$ & 17 & $8,25 \%$ \\
\hline $33-36$ & 5 & $2,43 \%$ \\
\hline$>37$ & 1 & $0,49 \%$ \\
\hline Total & 206 & $100,00 \%$ \\
\hline \multicolumn{3}{|l|}{ Cor } \\
\hline Parda & 106 & $51,46 \%$ \\
\hline Branca & 63 & $30,58 \%$ \\
\hline Negra & 32 & $15,53 \%$ \\
\hline Não quiseram responder & 5 & $2,43 \%$ \\
\hline Total & 206 & $100,00 \%$ \\
\hline \multicolumn{3}{|l|}{ Estado civil } \\
\hline Solteira & 179 & $86,89 \%$ \\
\hline Casada & 20 & $9,71 \%$ \\
\hline Divorciada & 3 & $1,46 \%$ \\
\hline União Estável & 3 & $1,46 \%$ \\
\hline Não quis responder & 1 & $0,49 \%$ \\
\hline Total & 206 & $100,00 \%$ \\
\hline
\end{tabular}

Fonte: Ramos TLFC, et al., 2021.

No que diz respeito às informações recebidas pelas participantes da pesquisa sobre neoplasia maligna da mama, 171 afirmaram ter recebido (83\%), como mostra o Gráfico 1.

Gráfico 1 - Distribuição do número de estudantes de uma Universidade Federal do Nordeste que receberam informação sobre neoplasia maligna da mama nos últimos dois anos.

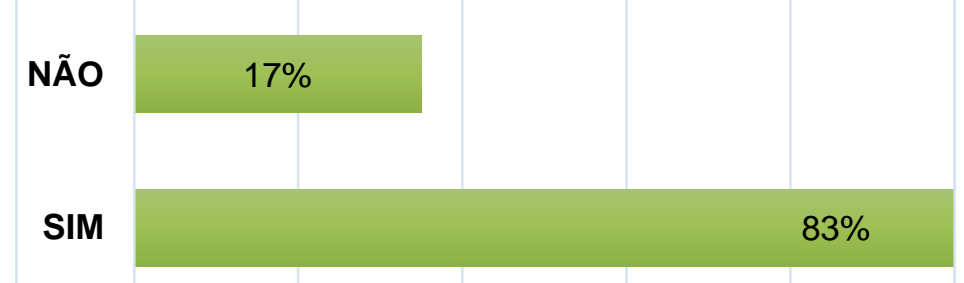

Fonte: Ramos TLFC, et al., 2021. 
No que corresponde aos locais por meio dos quais as participantes receberam essas informações, o questionário possibilitou a marcação de mais de uma alternativa, onde 61 estudantes $(29,6 \%)$ marcaram a alternativa correspondente apenas aos meios de comunicação, enquanto 32 estudantes (15,5\%) marcaram que obtiveram informações através dos meios de comunicação e amigos, familiares e conhecidos, como mostra a Tabela 2.

Tabela 2 - Distribuição do número de estudantes de uma Universidade Federal do Nordeste com relação aos locais por onde receberam informação sobre neoplasia maligna da mama.

\begin{tabular}{lcc}
\hline Meios de informação & (N) & (\%) \\
\hline Meios de comunicação & 61 & 29,6 \\
Meios de comunicação e universidade & 13 & 6,3 \\
UBS, meios de comunicação, outros serviços de saúde e universidade & 1 & 0,5 \\
Amigos, familiares e conhecidos e universidade & 1 & 0,5 \\
UBS e universidade & 4 & 1,9 \\
UBS, meios de comunicação e universidade & 3 & 1,5 \\
Outros serviços de saúde e universidade & 2 & 1,0 \\
UBS, meios de comunicação e amigos, familiares e conhecidos & 2 & 1,0 \\
Meios de comunicação e UBS & 5 & 2,4 \\
Meios de comunicação, outros serviços de saúde e universidade & 2 & 1,0 \\
UBS, meios de comunicação e outros serviços de saúde & 1 & 0,5 \\
Universidade & 19 & 9,2 \\
Meios de comunicação, amigos, familiares e conhecidos e outros & 1 &, 5 \\
serviços de saúde & 1 & 0,5 \\
UBS e amigos, familiares e conhecidos & 1 & 1,9 \\
Amigos, familiares e conhecidos & 4 & 3,9 \\
UBS & 8 & 2,9 \\
Outros serviços de saúde & 6 & 1,0 \\
Não lembra & 2 & 17,0 \\
Não recebeu & 35,5 \\
Meios de comunicação e amigos, familiares e conhecidos & 35 & 1,5 \\
Meios de comunicação e outros serviços de saúde & 32 & 100,0 \\
\hline Total & 3 & 206 \\
\hline
\end{tabular}

Fonte: Ramos TLFC, et al., 2021.

No tocante ao conhecimento repassado pela Universidade sobre neoplasia maligna da mama, apenas o curso de Enfermagem demonstra ter um maior conhecimento, onde 27 estudantes (79\%) responderam que se sentem bem informadas, entretanto, o curso de Matemática obteve o pior índice, com 35 estudantes (97\%) que responderam não ter acesso à informação sobre esse assunto dentro do Campus universitária, como evidencia o Gráfico 2.

Gráfico 2 - Distribuição do número de estudantes de seis cursos de graduação de uma Universidade Federal do Nordeste com relação ao nível de conhecimento sobre neoplasia maligna da mama.

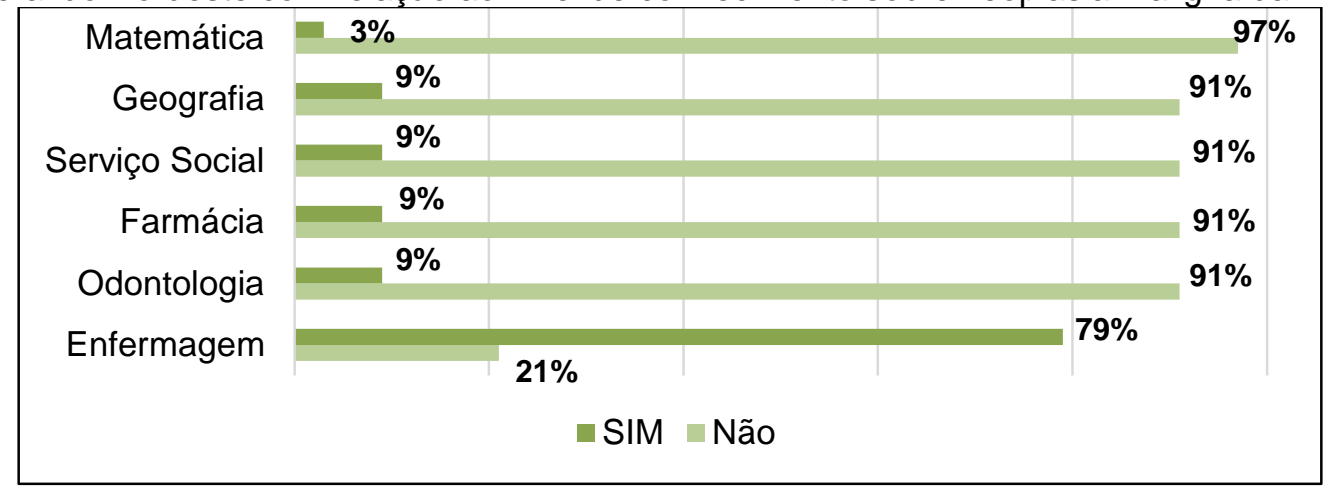

Fonte: Ramos TLFC, et al., 2021. 


\section{DISCUSSÃO}

A neoplasia maligna da mama configura-se na formação de um tumor maligno como consequência da multiplicação rápida de células anormais, podendo manifestar-se por meio de variadas formas clínicas e morfológicas. É relativamente raro antes dos 35 anos, contudo, maior que esta faixa etária sua incidência aumenta rápida e gradativamente acarretando em mudanças físicas e emocionais importantes nas mulheres (INCA, 2015).

É o segundo tipo de neoplasia mais recorrente no mundo e o que mais acomete as mulheres, correspondendo a $22 \%$ dos casos novos a cada ano. Esta doença é considerada uma grande e grave problema de saúde pública, consequentemente é uma das mais importantes causas de morbidade e mortalidade na mulher (ARÊDES TB, et al., 2015).

Nesse estudo, a faixa etária prevalente foi dos 18 aos 22 anos, o que sugere um maior acesso ao ensino superior, informação e, consequentemente, menor risco de desenvolver neoplasia maligna da mama. Sendo este último evidenciado numa pesquisa indicando que a ocorrência de neoplasia maligna da mama em mulheres jovens é baixa (DUGNO MLG, et al., 2014). Outro estudo também ressalta essa afirmação, que a neoplasia maligna da mama é rara antes dos 35 anos, possuindo uma alta incidência até os 50 anos e após esta idade, seu progresso ocorre de forma lenta. No entanto, essa faixa etária apresenta discreto aumento nesse público, evidenciando a importância do rastreamento precoce (DAGMAR SL, et al., 2014; SOUZA NHA, et al., 2017).

Ainda assim, o número de casos aumentou à medida que a idade das mulheres também aumentava, ocorrendo predomínio na faixa etária entre 36 e 39 anos. Algumas pesquisas concordam com tal achado, onde a incidência aumenta entre 15 e 39 anos de idade, além de que a maioria das pacientes jovens com neoplasia maligna da mama situava-se na faixa etária entre 35 e 39 anos (PINHEIRO AB, et al., 2013).

Portanto, o fato de existir a preconização da faixa etária para realização da mamografia, falta de informação sobre fatores de risco, falta de acesso aos serviços de saúde especializados, leva ao atraso no diagnóstico em muitas mulheres jovens, consequentemente, poderia estar contribuindo para a descoberta da doença em um estágio mais avançado (PEREIRA HFBESA et al. 2017).

Em relação à cor/raça das participantes, grande parte se autorreferiram pardas. Numa pesquisa sobre este assunto foi conhecido que a variável "raça/cor" foi introduzida pelo Sistema de Informação sobre Mortalidade (SIM) em 1995 e desde então ocorreu aumento da sua ligação com as desigualdades na saúde do Brasil. No entanto, a busca por essa informação se mostra deficiente, já que a busca recomendada por essa informação é a autoclassificação. No momento, a grande concentração da população negra encontra-se no Nordeste (SOARES LR, et al., 2015; ARTES A, 2018).

Ainda sobre a cor/raça, outro estudo demonstrou que a grande maioria das mulheres jovens com neoplasia maligna da mama declarou ser branca ou parda, corroborando que a incidência de neoplasia maligna da mama em mulheres jovens diversifica de acordo com a raça, pois mulheres negras com idade menor que 35 anos são responsáveis pelo dobro da incidência de neoplasia maligna da mama invasivo e o triplo da mortalidade, quando comparadas a mulheres brancas (PINHEIRO AB, et al., 2013; ASSIS EA, et al.2019).

Também foi analisado nessa pesquisa o estado civil, em que a maioria das participantes é solteira o que contrapõe outro estudo o qual afirma que a grande maioria das mulheres do seu estudo eram casadas ou viviam com um companheiro, destacando que o estado conjugal não é considerado um fator de risco para o desenvolvimento da doença, no entanto, a circunstância "de ter um companheiro está associada a um melhor suporte social, otimismo e qualidade de vida entre mulheres sobreviventes (HADDAD NC, et al., 2015; FARINA A, et al. 2017).

Outro estudo com mulheres norte-americanas apontou que o risco de desenvolvimento de neoplasia maligna da mama em estágio avançado cresceu cerca de três vezes nas mulheres que nunca foram casadas (DUGNO MLG, et al., 2014). Desse modo, é sugestivo que o companheirismo de um relacionamento afetivo aumente as chances da mulher exercer um maior autocuidado, além de ter suporte emocional do diagnóstico 
ao tratamento, nos casos confirmados de neoplasia maligna da mama, além de ajuda nas atividades de vida diária, já que no período da doença a mulher precisa se readaptar ao seu estilo de vida, ao que costumava fazer diariamente, já que inicialmente ela pode estar debilitada e sentindo-se desnecessária no cotidiano de vida que possui (ALMEIDA TG, et al., 2015).

No que diz respeito às informações adquiridas sobre neoplasia maligna da mama, a maioria das participantes tiveram acesso à informação sobre a doença através dos "meios de comunicação", o que pode estar atrelado ao fácil acesso dessas mulheres aos meios de informação, como rádio, TV e Internet. As campanhas publicitárias governamentais relacionadas à saúde e veiculadas nos meios de comunicação têm a finalidade de intervir nas decisões das pessoas em prol do bem-estar social e são conhecidas como campanhas de marketing social, com foco nas mudanças de hábitos da população (MARTINS AFH, et al., 2015).

Ademais, o governo federal fez o investimento de seis milhões de reais em 2011 e 2012, além de dezoito milhões até 2014, em comunicação para propagação de informação sobre a prevenção da neoplasia maligna da mama, como a realização da mamografia e o autoexame das mamas. No entanto, com todo esse investimento, as taxas de mortalidade no país continuam altas, e a incidência estimada para o ano de 2014 foi em torno de 57.120 mil novos casos, o que significa a necessidade de avaliação mais afundo sobre a eficácia dessas campanhas em atingir e modificar o comportamento do seu público alvo (MARTINS AFH, et al., 2015).

Logo, é necessário que, além de existir uma avaliação dessas propagandas, que ocorra também uma continuidade delas, e não apenas no mês de conscientização do problema, que acontece todos os anos em outubro. Também é fundamental criar espaços de diálogo em todos os níveis de atenção para que as mulheres tirem suas dúvidas a respeito do problema. Esses locais podem ser escolas, creches, a própria universidade, Centros de Referências de Assistência Social (CRAS), Unidades Básicas de Saúde (UBS) e até mesmo os hospitais.

Outro fator importante é o autoexame das mamas, onde a maioria afirmou ter recebido essa informação, que está ligada às propagandas nos meios de comunicação. Todavia, a maioria das participantes não o realiza, podendo acontecer devido à falta de orientação correta nos meios de saúde. Segundo uma dada pesquisa, grande parte dos estudos nacionais demonstra conhecimento feminino sobre o autoexame das mamas (AEM). Contudo, esse saber se diferencia de acordo com as regiões, onde o Nordeste tem o maior índice de mulheres na faixa etária dos 40 aos 44 anos que declaram conhecer o AEM. Porém, sua indicação é controversa. Contudo, a mídia leiga ainda é a principal fonte dessa informação. A ocorrência dos jovens não realizarem o AEM em comparação com as mulheres com mais de 45 anos é que, na visão deles, a neoplasia maligna da mama é uma doença improvável de acontecer na faixa etária jovem em comparação com a faixa etária mais avançada (RODRIGUES TCGF, et al., 2016).

Ainda segundo os mesmos autores, a pesquisa demonstrou que a maioria dos participantes de todas as faixas etárias não se considera apta a diferenciar o tecido mamário normal de um possível nódulo. $\mathrm{E}$ além disso, a descoberta de alterações mamárias gera sentimentos negativos na mulher, em virtude da aflição da possibilidade de ser uma neoplasia maligna e de cirurgias mutiladoras. Dessa forma, a descoberta de alguma alteração pode fazer com que as mulheres prorroguem a consulta com o especialista e prolonguem atraso no diagnóstico precoce (RODRIGUES TCGF, et al., 2016).

Por outro lado, o AEM tem importância significativa no diagnóstico precoce da neoplasia da mama, demonstrando pequenas lesões e menor número de linfonodos axilares comprometidos pela neoplasia. Porém, o AEM deve ser orientado quanto à conscientização feminina sobre as ações contra a neoplasia maligna da mama, principalmente em locais onde o acesso ao atendimento primário é precário. $O$ Ministério da Saúde recomenda ao SUS a realização de atividades que envolvam o ensino da palpação das mamas pela própria mulher como mecanismo do autocuidado. (SILVA RM, et al., 2015).

Portanto, é necessário orientar essas mulheres nos diversos meios da sociedade, de como se realiza esse autoexame, a faixa etária para tal e os fatores de risco envolvidos para essa avaliação. Para isso, é essencial 
metas e parcerias com os gestores municipais, estaduais e a universidade para criação de projetos que levem informações à sociedade em geral, por meio de escolas, creches, indústrias e todos os locais que possam receber informações acerca da doença.

Já no que concerne ao conhecimento das participantes sobre neoplasia maligna da mama repassado pela universidade, dos seis cursos entrevistados, apenas o de Enfermagem obteve maior número de participantes que relataram possuir esse conhecimento vindo da própria instituição, o que pode sugerir um maior contato desses estudantes aos serviços de saúde por meio de atividades práticas supervisionadas, além disso, esse assunto é tema em disciplinas da graduação; opondo-se ao pior resultado dos seis cursos, que foi o de Matemática, onde grande parte das participantes entrevistadas responderam que não têm acesso às informações sobre neoplasia maligna da mama dentro do campus, podendo ser pela falta de atuação da universidade em campanhas que informem sobre o assunto, além da falta de projetos de extensão que atuem dentro da universidade e não apenas fora dos muros do campus.

A universidade constitui espaços sociais estratégicos para a promoção da saúde de grupos populacionais específicos, como é o caso dos estudantes, e sobre a população em geral. A promoção da saúde desses grupos acarreta disponibilizar as condições necessárias para melhorar e exercer controle sobre sua saúde (BRANDÃO MGSA, et al., 2017; SACRAMENTO RC, et al. 2020).

O envolvimento da universidade com projetos de promoção da saúde ou prevenção de agravos, como é o caso da disseminação de conhecimento acerca da neoplasia maligna da mama, pode obter muitas vantagens, desde o reconhecimento de sua imagem pública, sua relevância para a saúde da sua cidade, estado e país, além da melhoria dos projetos institucionais e pedagógicos, incluindo a melhoria da qualidade de vida dos envolvidos, e as condições de atividade e de permanência das pessoas que ali trabalham, estudam, vivem e socializam (MAIRINK APAR, et al., 2020; OLIVEIRA DAL, et al., 2020).

A universidade é um local de aprendizagem e desenvolvimento, apoiada no tripé ensino-pesquisaextensão, de modo que deveria caracterizar-se como um ambiente onde os estudantes desenvolvessem habilidades e propagassem seus conhecimentos com toda comunidade acadêmica. Por isso, é essencial que se estabeleça atividades de informação voltadas ao meio acadêmico de todos os cursos, visto que até os cursos relacionados à saúde informaram não ter conhecimento suficiente sobre o assunto, o que indica uma contradição, já que existe a falsa impressão de que esses cursos dominam esses assuntos, principalmente os de utilidade pública, como é o caso da neoplasia maligna da mama.

\section{CONCLUSÃO}

Os estudantes receberam informações sobre neoplasia da mama, porém não foi a universidade que proporcionou este conhecimento, sendo o conhecimento obtido por meios de comunicação, como rádio e TV. Logo, a partir desses dados, é importante repensar as práticas de educação e propagação do conhecimento, além de ser imprescindível que a universidade invista em meios de transformação através de estratégias que levem à promoção da saúde e formem cidadãos que serão propagadores de conhecimento, além de democratizar o acesso à informação no meio universitário. Espera-se que os estudantes dos cursos da área de saúde se apropriem deste tema e de outros, pois são eles que atuaram diretamente com a educação em saúde, levando informações que possa contribuir para prevenção e promoção dos agravos à saúde.

\section{REFERÊNCIAS}

1. ALMEIDA TG, et al. Vivência da mulher jovem com câncer de mama mastectomizada. Esc Anna Nery, 2015; 19(3): 432-438.

2. ARÊDES TB, et al. Nível de informação e qualidade de vida em mulheres após o câncer de mama. SALUSVITA, Bauru, 2015, 34(2): 291-306.

3. ARTES, A. Dimensionando as desigualdades por sexo e cor/raça na pós-graduação brasileira. Educação em Revista. $2018 ; 34 ; 1-23$.

4. ASSIS EA, et al. Perfil sociodemográfico do câncer de mama na Bahia nos anos de 2013 a 2018. RevistaTextura, 2019; 13(21): 104-113.

5. BARRETO-NETO NJS, et al. Perfil epidemiológico dos subtipos moleculares de carcinoma ductal da mama em população de pacientes em Salvador, Bahia. Rev Bras Mastologia, 2014, 4(24): 98-102. 
6. BARTA SK, et al. Changes in the influence of lymphoma- and HIV-specific factors on outcomes in AIDS related NonHodgkin Lymphoma. Annals of Oncology, Dordrecht, 2015, 26(5): 958-966.

7. BRANDÃO MGSA, et al. Ultrapassando os muros da universidade: a monitoria acadêmica como ferramenta de educação em saúde. Raízes e Rumos, 2017, 5(2): 151-159.

8. DAGMAR SL, et al. Câncer de mama: estudo caso controle no Sul do Brasil. Revista Ciência \& Saúde, jan-abr 2014, 1(7): 19-26.

9. DUGNO MLG, et al. Perfil do câncer de mama e relação entre fatores de risco e estadiamento clínico em hospital do Sul do Brasil. Revista Brasileira de Oncologia Clínica, 2014, 36(10): 60-66.

10. FARINA A, et al. Perfil epidemiológico, clínico, anátomo patológico e imunohistoquímico das pacientes com câncer de mama em Cuiabá (MT). Rev Bras Mastologia. 2017;27(1):74-9.

11. GARCIA SN, et al. Os domínios afetados na qualidade de vida de mulheres com neoplasia mamária. Rev Gaúcha Enferm, 2015, 2(36): 89-96.

12. HADDAD NC, et al. Perfil sociodemográfico e de saúde de mulheres submetidas à cirurgia para câncer de mama. Revista Hospital Universitário Pedro Ernesto, 2015; 14 (Supl 1): 28-35.

13. INCA. Instituto Nacional do Câncer José de Alencar Gomes da Silva. Estimativa 2016: incidência de câncer no Brasil. Rio de Janeiro: INCA; 2015.

14. INCA. Instituto Nacional do Câncer José de Alencar Gomes da Silva. Estimativa 2020: incidência de câncer no Brasil. Rio de Janeiro: INCA; 2019.

15. JEMAL A, et al. O atlas do câncer. The Cancer Atlas. 2nd Ed. Atlanta, GA: American Cancer Society; 2014.

16. LEITE MAC, et al. Avaliação da autoestima em pacientes oncológicos submetidos a tratamento quimioterápico. Rev. Latino-Am. Enfermagem, 2015, 6(23): 1082-1089.

17. MAIRINK APAR, et al. Educação em saúde para estudantes sobre câncer de mama: relato de experiência. Revista UFG, 2020, 20(26): 1-18.

18. MARTINS AFH, et al. Análise da campanha Outubro Rosa de prevenção do câncer de mama em Viçosa, MG. Revista de Ciências Humanas, 2014; 2(14): 539-556.

19. MIGOWSKI A, et al. Diretrizes para detecção precoce do câncer de mama no Brasil. III - Desafios à implementação. Cadernos de Saúde Pública. 2018; 34(6): 1-14.

20. OLIVEIRA DAL, et al. Autocuidado e prevenção do câncer de mama: conhecimento das estudantes de graduação em saúde. REAS, 2020, 12(10): 1-8.

21. OLIVEIRA MF, et al. Estudo retrospectivo de pacientes diagnosticados com câncer de mama internados em hospital universitário. Rev Bras Mastologia. 2016; 26(2):56-9.

22. PEREIRA HFBESA, et al. Aspectos Clínicos e Patológicos do Câncer de Mama em Mulheres Jovens Atendidas na FCecon entre 2003 e 2013. Revista Brasileira de Cancerologia 2017; 63(2): 103-109

23. PINHEIRO AB, et al. Câncer de Mama em Mulheres Jovens: Análise de 12.689 Casos. Rev. Brasileira de Cancerologia, 2013; 59(3): 351-359.

24. PROLLA CMD, et al. Conhecimento sobre câncer de mama e câncer de mama hereditário entre enfermeiros em um hospital público. Rev. Latino-Am. Enfermagem, jan.-fev 2015; 23(1):90-97.

25. RODRIGUES TCGF, et al. Conhecimento de 820 mulheres atendidas no Hospital Universitário da Universidade Federal de Juiz de Fora sobre autoexame das mamas. Rev Bras Mastologia. 2016, 2(26): 60-4.

26. SACRAMENTO RC, et al. Ações de Enfermagem na promoção da saúde da mulher no contexto Amazônico. Research, Society and Development. 2020; 9(5): 1-10.

27. SILVA RM, et al. Educação em saúde para prevenção do câncer de mama no município de Piripiri-PI: atuação do petsaúde. R. Epidemiol. Control. Infec, 2015, 4(5): 203-205.

28. SOARES LR, et al. Mortalidade por câncer de mama feminino no Brasil de acordo com a cor. Rev Bras Ginecol Obstet. 2015; 37(8):388-92.

29. SOUZA NHA, et al. Câncer de mama em mulheres jovens: estudo epidemiológico no nordeste brasileiro. SANARE, Sobral, 2017;16(02): 60-67.

30. STEWARD BW, WILD CP. World Cancer Report 2014. Lyon: International Agency for Research on Cancer, Geneva: World Health Organization, 2014. 